Cite this: Analyst, 2013, 138, 6881

Received 5th August 2013

Accepted 10th September 2013

DOI: 10.1039/c3an01478a

www.rsc.org/analyst

\section{Phytochemical analyses of Ziziphus jujuba Mill. var. spinosa seed by ultrahigh performance liquid chromatography-tandem mass spectrometry and gas chromatography-mass spectrometry}

\author{
Bao Yang, ${ }^{a}$ Hongshun Yang, ${ }^{b}$ Feng Chen, ${ }^{c}$ Yanglin Hua ${ }^{d}$ and Yueming Jiang ${ }^{* a}$ \\ Ziziphus jujuba Mill. var. spinosa (Z. jujuba) seeds have attracted much attention within the field of \\ medicine due to their significant effects against disturbances of the central nervous system. Secondary \\ metabolites composition is key to the influence of the pharmaceutical and commercial qualities of this \\ plant. In this work, the phytochemical profile of $Z$. jujuba seeds was analysed by ultrahigh performance \\ liquid chromatography-tandem mass spectrometry (UPLC-MS/MS) and gas chromatography-mass \\ spectrometry (GC-MS). The UPLC-MS/MS information identified the main secondary metabolites in $Z$. \\ jujuba seeds, including flavonoid C-glycosides, triterpene acids and unsaturated fatty acids. The leading \\ chemical identified by UPLC-MS/MS was betulinic acid, and oleic acid was the leading volatile from the \\ GC-MS results. All the samples tested showed similar phytochemical profiles, but levels of the chemical \\ compounds varied. Principal component analysis revealed the principal secondary metabolites that \\ could define the differences in quality. It was confirmed that the combination of UPLC-MS/MS and GC- \\ MS was an effective technique to demonstrate the pharmaceutical quality of $Z$. jujuba seeds.
}

\section{Introduction}

Ziziphus is a genus which has approximately 40 species of the Rhamnaceae family, and is distributed mainly in warm and subtropical regions throughout the world. As an important economic species of this genus, Zizyphus jujuba Mill. var. spinosa has generated much commercial value due to its significant pharmacological functions. Insomnia and anxiety are the main pharmaceutical activities attributed to the species in the wider application of this medicinal species. ${ }^{\mathbf{1}}$ In addition, it also has good bioactivities against weakness, hyperhidrosis, liver complaints, urinary trouble, fever, hypertension and hyperlipidemia. $^{2,3}$ Compared to other hypotonic plants, such as kava, ${ }^{4}$ Z. jujuba (seeds) has a slow onset of effect, and has both a longterm effect on sleep architecture and low toxicity. As the commercial quality of the biological sources is readily affected by a number of factors, like the growing environment, processing conditions and genetic background the pharmaceutical capabilities of $Z$. jujuba seeds from various commercial sources vary.

${ }^{a}$ Key Laboratory of Plant Resources Conservation and Sustainable Utilization, South China Botanical Garden, Chinese Academy of Sciences, Guangzhou 510650, China. E-mail:ymjiang@scbg.ac.cn

${ }^{b}$ Food Science and Technology Programme, Department of Chemistry, National University of Singapore, Singapore 117543, Singapore

${ }^{c}$ Department of Food Science and Human Nutrition, Clemson University, Clemson, SC 29634, USA

${ }^{d}$ Infinitus (China) Company Ltd, Guangzhou 510665, China
Therefore, the pharmaceutical quality needs to be determined before use.

Natural bioactive compounds, including phenolics and carbohydrates, are responsible for the pharmaceutical activities of this medicinal herb. ${ }^{5,6}$ As an important ingredient of Chinese traditional medicines, $Z$. jujuba seeds have abundant bioactive secondary metabolites, including triterpene acids, unsaturated fatty acids and flavonoid C-glycosides, ${ }^{7-9}$ which are critical chemicals involved both in disease treatment and health benefits.

Metabolomics is being increasingly utilized to gain insight into the chemical composition of biological materials. ${ }^{\mathbf{1 0}}$ Determination of metabolomic profiles by ultrahigh performance liquid chromatography-tandem mass spectrometry (UPLC-MS/ MS) and gas chromatography-mass spectrometry (GC-MS) has been recently adopted as an effective technique to understand the chemical nature of a medicinal plant. Though nuclear magnetic resonance spectroscopy (NMR) is also widely applied in metabolomics, the MS-based technique shows better precision and resolution than NMR. Therefore, in this work, the phytochemical profile of $Z$. jujuba seed was analysed by both UPLC-MS/ MS and GC-MS to judge the quality of commercial Z. jujuba seed.

\section{Materials and methods}

\subsection{Plant material}

Six $Z$. jujuba seeds were purchased from Neautus Traditional Chinese Medicine Co. Ltd (Chengdu, China), Guanghong 
Pharmaceutical Co. Ltd (Guangzhou, China), and Yifang Chinese Medicine Co. Ltd (Anguo, China), respectively. Seed samples 2\#, 4\# and 6\# were raw Z. jujuba seeds from Wenshang, Shandong province, Zhanhuang, Hebei province and Anguo, Hebei province, respectively. They were treated to obtain samples $1 \#, 3 \#$ and $5 \#$ by the following procedures. One kilogram of raw $Z$. jujuba seeds was heated at $200{ }^{\circ} \mathrm{C}$ with agitation. The seeds were toasted brown and when a pleasant aroma could be smelled, they were collected as the heat-treated sample. Each sample was pulverized into powder and sieved through 60-mesh sieve.

\subsection{UPLC-MS/MS analysis}

Two grams of $Z$. jujuba seed powder were extracted with $20 \mathrm{ml}$ methanol during the course of one week in the dark at room temperature. The extract was centrifuged at $8000 \mathrm{~g}$ for $10 \mathrm{~min}$. Each sample was extracted in triplicate. The supernatant was collected and subjected to UPLC-MS/MS analysis. Acquity ${ }^{\mathrm{TM}}$ UPLC (Waters, Milford, MA, USA) equipped with an Acquity ${ }^{\mathrm{TM}}$ UPLC BEH C18 column $(1.7 \mu \mathrm{m}, 2.1 \times 150 \mathrm{~mm})$ was used for chemical isolation. A gradient elution programme was conducted as follows: $0-2 \mathrm{~min}, 5 \%$ solvent $\mathrm{B}$; $2-17 \mathrm{~min}$, from $5 \%$ to $30 \%$ solvent B; $17-20 \mathrm{~min}$, from $30 \%$ to $55 \%$ solvent $\mathrm{B} ; 20-35$ min, from $55 \%$ to $100 \%$ solvent B; $35-37 \mathrm{~min}$, from $100 \%$ to $5 \%$ solvent B; 37-39 min, 5\% solvent B. Solvent A was $0.1 \%$ formic acid in water, and solvent $\mathrm{B}$ was acetonitrile. The flow rate was $0.25 \mathrm{ml} \min ^{-1}$. The column temperature was kept at $35{ }^{\circ} \mathrm{C}$. Detection was performed on a triple quadrupole mass spectrometer detector using an electrospray ionization (ESI) interface. Ionization of the analytes was achieved using ESI in negative mode. The interface conditions were as follows: capillary voltage, $1.0 \mathrm{kV}$; cone voltage, $10 \mathrm{eV}$; collision voltage, $10 \mathrm{eV}$; source temperature, $150{ }^{\circ} \mathrm{C}$; desolvation temperature, 500 ${ }^{\circ} \mathrm{C}$; cone gas, nitrogen at a flow rate of $50 \mathrm{l} \mathrm{h}^{-1}$; desolvation gas, nitrogen at a flow rate of $800 \mathrm{l} \mathrm{h}^{-1}$; collision gas, argon at a flow

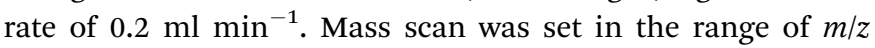
50-2000. The daughter ion mode was monitored at a collision voltage in the range of $10-40 \mathrm{eV}$.

\subsection{GC-MS analysis}

One gram of $Z$. jujuba seed powder was extracted with $10 \mathrm{ml}$ of hexane-acetone $(7: 3, v / v)$ during the course of one week in the dark at room temperature. The extracts were centrifuged at $8000 \mathrm{~g}$ for $15 \mathrm{~min}$. The supernatants were collected and subjected to trimethylsilyl derivatization due to the occurrence of organic acids. The trimethylsilyl derivatization was conducted by the same method as Yang et al. ${ }^{11}$ A gas chromatography-mass spectrometer (GCMS-QP 2010, Shimadzu, Kyoto, Japan) was used to analyse the chemical composition. The derivatives were loaded into a RTX-5 capillary column. The temperature program was set as follows: the initial temperature of column was $50{ }^{\circ} \mathrm{C}$, holding for $1 \mathrm{~min}$, increasing to $250{ }^{\circ} \mathrm{C}$ at $3{ }^{\circ} \mathrm{C} \mathrm{min}{ }^{-1}$, holding for $17 \mathrm{~min}$, increasing to $280{ }^{\circ} \mathrm{C}$ at $10{ }^{\circ} \mathrm{C} \mathrm{min}^{-1}$; injection temperature: $250{ }^{\circ} \mathrm{C}$. The ion source of the mass spectrometer was set at $250{ }^{\circ} \mathrm{C}$. The scanning $\mathrm{m} / \mathrm{z}$ range was $20-550$ amu. $1 \mu \mathrm{l}$ of sample was injected and the split ratio was $10: 1$. The carrier

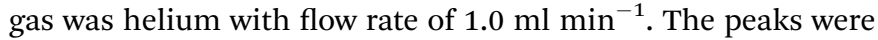
identified by NIST database and retention index.

\subsection{Data analysis}

For each sample, the peak heights of chemicals in the mass spectra were recorded and averaged over three replicates. An unsupervised multivariate statistical method, principal component analysis (PCA), was used on UV-scaling data. PCA was performed to visualize the clustering of different samples without any knowledge of their group membership.

\section{Results}

\subsection{The composition of semi-polar phytochemicals determined by UPLC-MS/MS}

UPLC-MS/MS analyses were conducted on the methanolic extract of $Z$. jujuba seeds to identify semi-polar chemicals. Fig. 1 shows the full scan ESI-MS spectra in negative mode, which record the levels of secondary metabolites. Identified chemicals are listed in Table 1 . Those chemicals with relatively high peak are interpreted here.

The leading peak was betulinic acid (retention time 30.55 min, Fig. 2). The MS spectra had an $[\mathrm{M}-\mathrm{H}]^{-}$at $m / z$ 455.0. The fragment ions were interpreted as follows: $\mathrm{m} / \mathrm{z} 437.0$ (loss of $\mathrm{H}_{2} \mathrm{O}$ ), 410.9 (loss of carboxyl). ${ }^{12}$ A base peak at $\mathrm{m} / \mathrm{z} 188.9$ was characteristic of the fragmentation of triterpenoid molecules with a lupane skeleton bearing a hydroxy group in position 3 . It had arisen from the fragmentation of the $\mathrm{C}$ ring by cleavage of C9-C11 and C8-C14 bonds followed by the loss of $\mathrm{H}_{2} \mathrm{O}^{13}$ Moreover, two peaks at $\mathrm{m} / \mathrm{z} 247.4$ and 202.6 were observed, which indicated the formation of the $\left[\mathrm{C}_{16} \mathrm{H}_{24} \mathrm{O}_{2}\right]$ fragment ion and further loss of carboxyl. ${ }^{14}$

The peak at 29.79 min showed an $[\mathrm{M}-\mathrm{H}]^{-}$at $m / z 454.9$ and an $[2 \mathrm{M}-\mathrm{H}]^{-}$at $m / z 911.1$, which indicated that the molecular weight (456) was equal to betulinic acid. The fragment ion at $\mathrm{m} / \mathrm{z}$ 411.4 was formed by the loss of the carboxyl moiety. $\mathrm{m} / \mathrm{z} 436.5$ indicated the loss of $\mathrm{H}_{2} \mathrm{O}$ due to the elimination of the hydroxyl group at position 3. Another two fragment ions at $m / z 207.2$ and 189.1 were detected. This fraction was identified as zizyphursolic acid based on above results and literature. ${ }^{15}$

Linoleic acid (molecular weight 280) was detected from the fraction at $32.06 \mathrm{~min}$, which had an $[\mathrm{M}-\mathrm{H}]^{-}$at $m / z$ 278.9. The fragment ion at $\mathrm{m} / \mathrm{z} 260.6$ was formed due to the loss of $\mathrm{OH}$ in the carboxyl group. $\mathrm{m} / \mathrm{z} 58.7$ represented the occurrence of acetic acid. A peak at $m / z 70.6$ was detected.

Spinosin, a flavonoid C-glycoside, is a characteristically bioactive compound in $Z$. jujuba seeds, which was detected at $11.78 \mathrm{~min}$. The mass spectra showed an $[\mathrm{M}-\mathrm{H}]^{-}$at $\mathrm{m} / \mathrm{z} 606.7$ and a $[2 \mathrm{M}-\mathrm{H}]^{-}$at $m / z 1214.9$ for the precursor ion. The fragment ion at $m / z 427.2$ represented the loss of glucose and $\mathrm{H}_{2} \mathrm{O}$. The difference (120) between $\mathrm{m} / \mathrm{z} 606.7$ and 486.8 was characteristic of the breakage of glucose at $C_{1}-\mathrm{O}$ and $C_{2}-C_{3}$. Other fragment ions were detected at $\mathrm{m} / \mathrm{z} 366.9$ and 118.8. They were characteristic of spinosin. This mass spectral information was confirmed in the literature. ${ }^{16}$ 

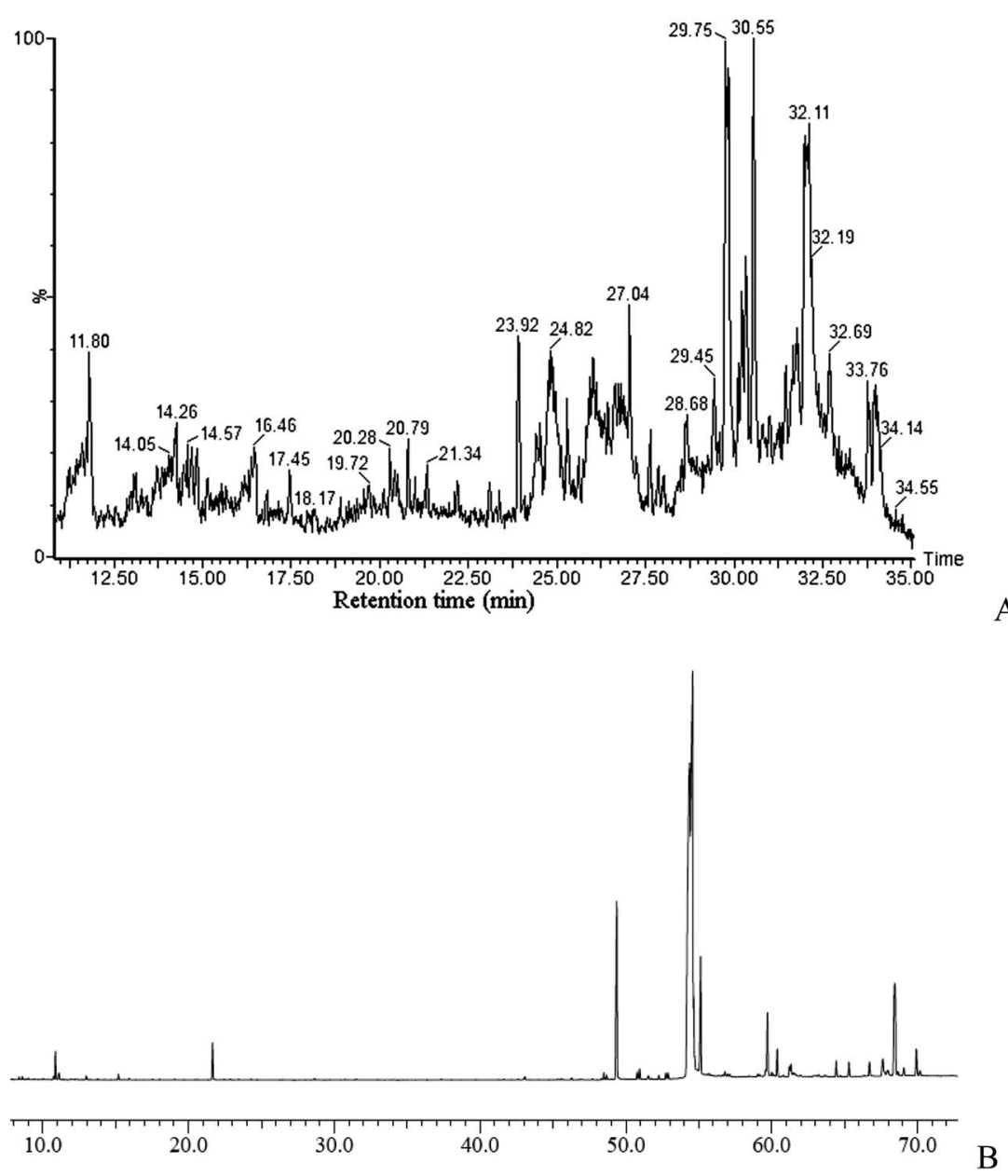

Fig. 1 UPLC-MS/MS and GC-MS profiles of Z. jujuba seeds. (A) Total ion chromatogram analysed by UPLC-MS/MS; (B) total ion chromatogram analysed by GC-MS.

Epiceanothic acid and ceanothic acid were detected at 27.07 and $23.92 \mathrm{~min}$, respectively. They had the same distribution of precursor and fragment ions. An $[\mathrm{M}-\mathrm{H}]^{-}$at $m / z 485.0$ and a $[2 \mathrm{M}-\mathrm{H}]^{-}$at $m / z 971.2$ were observed for the precursor ion. $\mathrm{m} / \mathrm{z}$ 471.4 was formed by the loss of methyl. Fragment ions at $\mathrm{m} / \mathrm{z}$ 441.0 was generated by the loss of one carboxyl moiety. $\mathrm{m} / \mathrm{z} 423.4$ indicated the loss of carboxyl and $\mathrm{H}_{2} \mathrm{O}$. In combination with the information in the literature, ${ }^{17}$ they were identified to be epiceanothic acid and ceanothic acid.

A peak at 20.79 min was analysed. An $[\mathrm{M}-\mathrm{H}]^{-}$at $m / z 1042.9$ was found to be the precursor ion, which was consistent with the molecular weight of jujuboside B (1044). The fragment ion at $\mathrm{m} / \mathrm{z} 910.9$ indicated the loss of xylose, and $\mathrm{m} / \mathrm{z} 310.6$ represented the occurrence of xylose-(1,2)-glucose. An $[\mathrm{M}-\mathrm{H}]^{-}$at $\mathrm{m} / \mathrm{z} 1205.1$ in the mass spectra at $20.28 \mathrm{~min}$ was consistent with the molecular weight of jujuboside A (1206). $\mathrm{m} / \mathrm{z} 1187.4$ was formed due to the loss of $\mathrm{H}_{2} \mathrm{O} . \mathrm{m} / \mathrm{z} 751.0$ indicated the pentasaccharide fragment ion.

\subsection{Volatile chemicals composition determined by GC-MS}

The volatile chemicals were extracted by hexane/acetone, trimethylsilylated and determined by GC-MS, which gave 22 volatile compounds (Table 2), including alcohols, carboxylic acids, esters, and terpenoids. The leading volatile chemical was oleic acid in the GC-MS profile (Fig. 1B). Linoleic acid and stearic acid were the next most prolific volatile chemical. Other volatile compounds with percentage higher than $5 \%$ were palmitic acid and 2-monoeicosanoylglycerol, respectively.

\subsection{Principal component analyses}

Principal component analysis was conducted on the semi-polar phytochemicals of $Z$. jujuba seed samples. The first two principal components explained $59.2 \%$ of the total variance, in which the first principal component explained $33.6 \%$ and the second explained $25.6 \%$. The loading plot (Fig. 3A) revealed the principal compounds responsible for the sample difference. As shown in Table 3, the chemical with an absolute value higher than 0.8 was the representative compound. The first principal component was represented by kaempferol 3-rutinoside, ceanothic acid, 6,8diglucopyranosyl-4', 5,7-trihydroxyflavanone, $3^{\prime} 5^{\prime}$-di-C-beta-D-glucosylphloretin, linoleic acid derivative and glycerol ester 1 . The second principal component was mainly characterized by $6^{\prime \prime}$ feruloylspinosin, jujuboside A, jujuboside B, a-nor-2,20(29)lupadiene-27,28-dioic acid and linoleic acid. The score plot showed the statistical similarity between samples. Sample $1 \# Z$. jujuba seeds was characterized by large amounts of linoleic acid 
Table 1 Semi-polar phytochemicals occurring in the methanolic extract of Z. jujuba seeds measured by UPLC-MS/MS

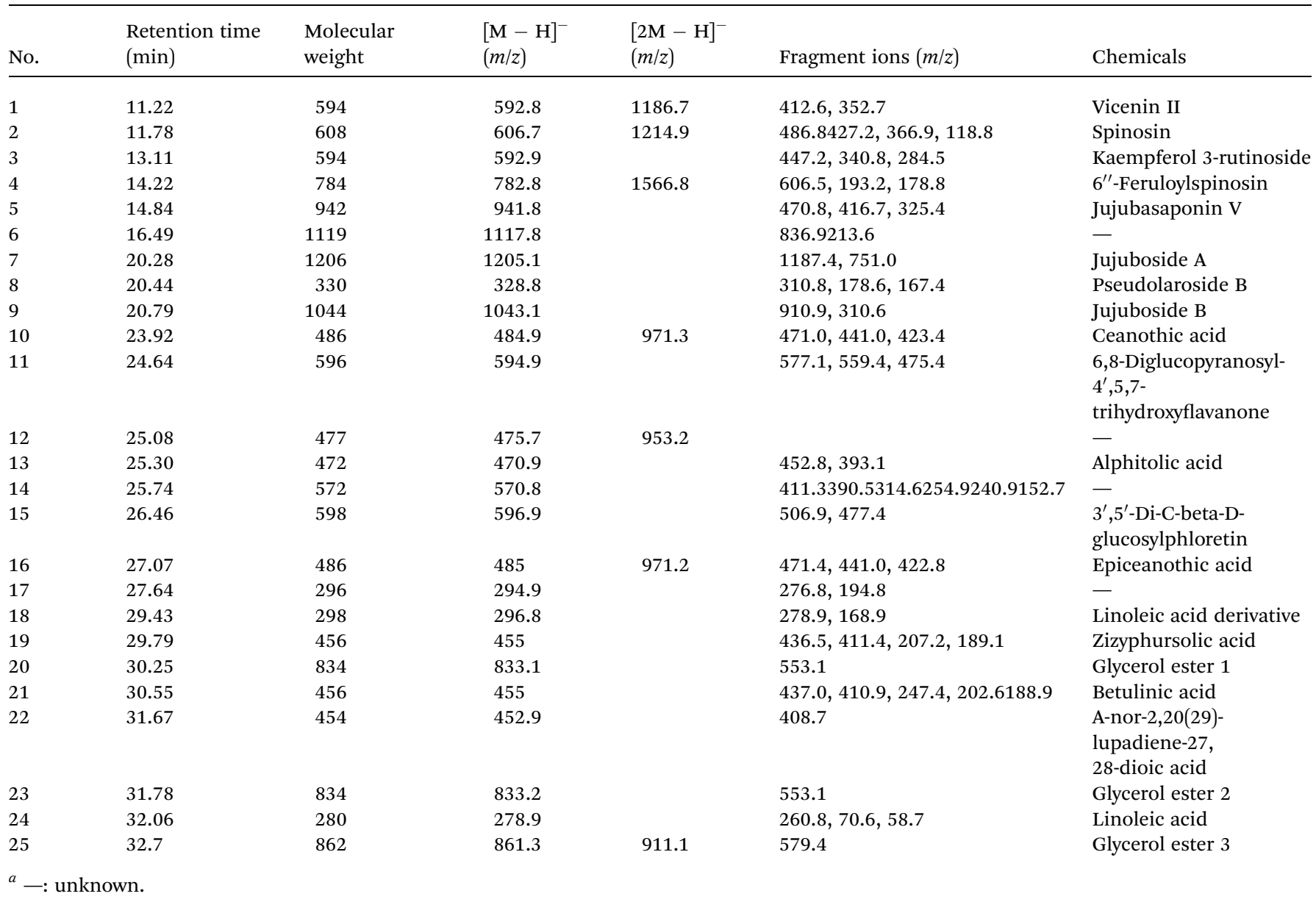

and low amount of $6^{\prime \prime}$-feruloylspinosin. Sample $2 \#$ and $4 \#$ seeds were located in the proximity of $1 \#$ seeds in the score plot (Fig. 3A), which indicated that they shared high similarity. Sample 3\# root showed high levels of pseudolaroside B and chemical 12, an unknown compound, and low levels of glycerol ester 1 . Sample $5 \#$ seeds was characterized by large amount of ceanothic acid. Sample 6\# was isolated from the other five seeds in the score plot, which suggested that it was quite different to them based on the chemicals levels observed.

Fig. 3B shows the results of PCA on volatile chemicals levels. The first two principal components explained $74.0 \%$ of the total variance, $52.1 \%$ by the first component and $21.9 \%$ by the second component. Major chemicals defining the first principal component were tetradecanoic acid, palmitic acid, linoleic acid methyl ester, oleic acid methyl ester, 9-octadecenoic acid, 11-eicosenoic acid and eicosanoic acid. The second principal component was mainly characterized by linoleic acid ethyl ester, ethyl oleate, 2-(9,12-octadecadienyloxy)-ethanol, 1-monopalmitoylglycerol and thymol-glucoside (Table 4). Samples 1\# and 6\# were isolated from the other four seeds as shown in the score plot (Fig. 3B). Sample 1\# Z. jujuba seeds was characterized by high levels of linoleic acid ethyl ester and thymol-glucoside. Sample 6\# seed was characterized by high levels of 11-eicosenoic acid and eicosanoic acid.

\section{Discussion}

\subsection{Phytochemical profile of $Z$. jujuba seeds}

Metabolomics is a systemic investigation of small molecular chemicals profiles, which provides a good way to evaluate the quality of plant resources, like medicinal herbs. At present, UPLC-MS/MS and GC-MS are two efficient and precise techniques to resolve the metabolomic profile of plant resources. ${ }^{\mathbf{1 8}}$ The former can identify semi-polar chemicals in a precise and time-saving manner. It has been widely applied to characterize plant secondary metabolites. GC-MS can reveal the chemical nature of volatile chemicals, which define the flavor of a plant. ${ }^{19}$ GC-MS also provides complementary data to UPLC-MS/MS analysis to obtain a complete metabolomic profile. From the phytochemical profile obtained in this work, it could be concluded that six $Z$. jujuba seeds had similar phytochemical profiles, but that the level of each peak varied.

Through phytochemical profile investigation, approximately 50 chemicals were identified from $Z$. jujuba seeds. As most of chemicals are difficult to obtain commercially, the structures identified in this paper identify the possible chemicals on the basis of fragment ions and the literature. It is generally accepted that $Z$. jujuba seeds are a good candidate to treat insomnia and anxiety. Thus, the distribution of pharmacological active 
<smiles>COc1cc2oc(-c3ccc(O)cc3)cc(=O)c2c(O)c1C1C(O)C(O)C(O)C(OC2OC(CO)C(O)C(O)C2O)C1O</smiles>

Spinosin<smiles>C=C(C)C1CCC2(C(=O)O)CCC3(C)C(CCC4C5(C)CCC(O)C(C)(C)C5CCC43C)C12</smiles>

betulinic acid<smiles>C=C1CCC2(C(=O)O)CCC3(C)C4CCC5C(CCC(O)C5(C)C)C4CCC3C2C1C</smiles>

Zizyphursolic acid<smiles>C=C(C)C1CCC2(C(=O)O)CCC3(C)C4CCC5C(C4CCC3C12)C(C(=O)O)C(O)C5(C)C</smiles>

epiceanothic acid

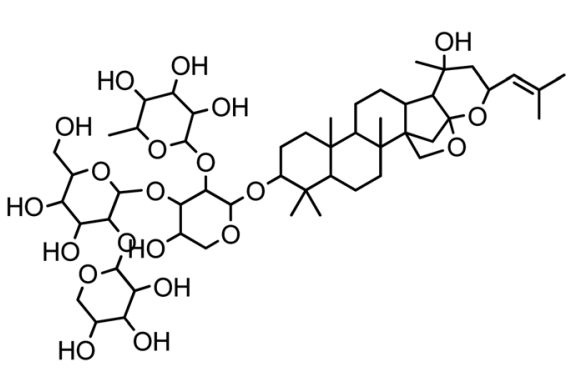

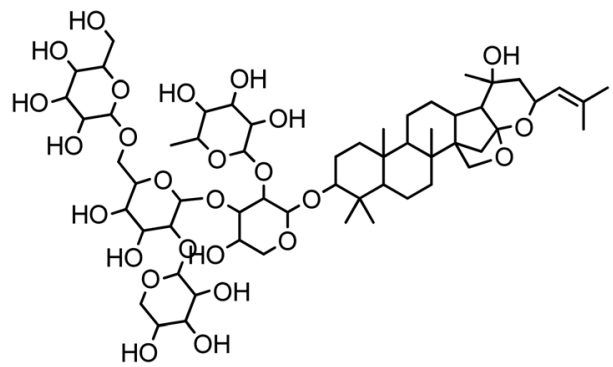

Jujuboside B

Fig. 2 Structures of characteristic chemicals in Z. jujuba seeds.

chemicals contributing to these effects should be investigated. Jujubosides, including jujubosides A and B, were detected as two independent peaks in the UPLC-MS/MS profile. Both contribute to the beneficial hypnotic effect of $Z$. jujuba seeds, and the mechanism of action for jujubosides is related to the serotonergic system. ${ }^{20}$ Spinosin is a characteristic flavonoid C-glycoside, which occurred at a high levels in $Z$. jujuba seeds (Fig. 1). It is another important bioactive compound responsible for the hypnotic effect of this plant. Similar to jujubosides, spinosin regulates the serotonergic system to inhibit insomnia. ${ }^{21}$ Further evidence suggested that spinosin potentiates pentobarbital-induced sleep via the serotonin-IA (5-hydroxytryptamine, 5-[HT.sub.1A]) receptor. ${ }^{22}$ Though cyclopeptide alkaloids have been reported as another pharmacologically active chemical to contribute to the hypnotic acitivity, ${ }^{23}$ they were not detected from the UPLC-MS/MS and GC-MS profiles in this work. P. tenuifolia root is another commonly used ingredient in traditional medicine to treat insomnia. ${ }^{24}$ The responsible active chemicals are oligosaccharide esters and saponins, which are different to the chemicals in Z. jujuba seeds. Therefore, it is important to define pharmacological quality by quantifying the levels of specific chemicals. UPLCMS/MS is a good choice of method to carry out the analysis.
In the UPLC-MS/MS profile, betulinic acid and bizyphursolic acid were the leading chemicals in $Z$. jujuba seeds. ${ }^{14}$ Both share similar structural characteristics as pentacyclic triterpene acid, which implies similar bioactivities. Comparing with zizyphursolic acid, betulinic acid is more common in the plant kingdom and has been proven to display diverse biological effects, including anticancer, antiinflammatory and antiviral activities. ${ }^{25}$ It is also a potential biological response modifier capable of strengthening the immune system of the host. ${ }^{26}$ The occurrence of these bioactive compounds in large amounts results in the good pharmacological and health-beneficial properties of $Z$. jujuba seeds.

GC-MS analysis showed that the major volatile compounds in $Z$. jujuba seeds were fatty acids. Among these, oleic acid was the leading volatile chemical, which is consistent with the determinations of Zhao et al. ${ }^{27}$ It is also a pharmacologically active chemical. The essential oil of $Z$. jujuba seeds has been reported to have hair growth promoting activity, ${ }^{28}$ and antiinflammatory activity. ${ }^{29}$

Based on the level and importance for pharmacological function, spinosin, zizyphursolic acid, betulinic acid, ceanothic acid, epiceanothic acid and linoleic acid identified in the UPLCMS/MS profile were selected to evaluate the quality of $Z$. jujuba 
Table 2 Volatile chemicals occurring in hexane-acetone extract of Z. jujuba seed determined by GC-MS

\begin{tabular}{|c|c|c|c|}
\hline No. & $\begin{array}{l}\text { Retention time } \\
\text { (min) }\end{array}$ & Chemicals & $\begin{array}{l}\text { Molecular } \\
\text { weight }\end{array}$ \\
\hline 1 & 10.87 & Octyl 3-alcohol & 130 \\
\hline 2 & 43.1 & Tetradecanoic acid & 228 \\
\hline 3 & 48.5 & Palmitelaidic acid & 254 \\
\hline 4 & 49.4 & Palmitic acid & 256 \\
\hline 5 & 50.8 & Linoleic acid methyl ester & 294 \\
\hline 6 & 50.9 & Oleic acid methyl ester & 296 \\
\hline 7 & 51.6 & 9-Octadecenoic acid & 282 \\
\hline 8 & 52.3 & Heptadecanoic acid & 270 \\
\hline 9 & 52.7 & Linoleic acid ethyl ester & 308 \\
\hline 10 & 53 & Ethyl oleate & 310 \\
\hline 11 & 54.4 & Linoleic acid & 280 \\
\hline 12 & 54.6 & Oleic acid & 282 \\
\hline 13 & 55.1 & Stearic acid & 284 \\
\hline 14 & 59.7 & 11-Eicosenoic acid & 310 \\
\hline 15 & 60.4 & Eicosanoic acid & 312 \\
\hline 16 & 61.2 & $\begin{array}{l}\text { 2-(9,12-Octadecadienyloxy)- } \\
\text { ethanol }\end{array}$ & 310 \\
\hline 17 & 64.4 & 1-Monopalmitoylglycerol & 330 \\
\hline 18 & 65.3 & Tetracosanoic acid & 368 \\
\hline 19 & 66.7 & Thymol-glucoside & 312 \\
\hline 20 & 67.6 & 2-Monooctadecenoylglycerol & 342 \\
\hline 21 & 68.5 & 2-Monoeicosanoylglycerol & 386 \\
\hline 22 & 69.9 & Squalene & 410 \\
\hline
\end{tabular}

seeds. Z. jujuba seeds with relatively high levels of bioactive compounds are considered good quality. Through ANOVA analysis of representative chemicals levels (data not shown), the results indicated that the six seed samples studied had no significant difference in the levels of zizyphursolic acid. The levels of betulinic acid $(2 \#>4 \#=6 \#)$, linoleic acid $(1 \#>6 \#)$, ceanothic acid $(3 \#=5 \#>6 \#>2 \#)$, epiceanothic acid $(5 \#>3 \#>$ $1 \#)$ were detected. It is not possible to define the total quality of Z. jujuba seeds through only the level of one bioactive compound. Therefore, PCA should be conducted to differentiate the quality between the samples.

\subsection{PCA of $Z$. jujuba seeds phytochemicals}

PCA is an unsupervised clustering technique for identifying patterns in data, expressing the data in such a way as to emphasize their similarities and differences. Through reducing the number of dimensions, it can define a limited number of principal components which describe independent variation in the results. ${ }^{10,30}$ In the present work, PCA was carried out on semi-polar and volatile chemicals of $Z$. jujuba seeds to find the principal phytochemicals defining the pharmacological quality. The loading plot displayed the influence of individual spectral peaks in each principal component and described the difference of the samples in the score plot. ${ }^{31}$ The points in the loading plot far from the zero point represented characteristic markers with the most confidence for each group. Fig. 3 shows the plots of PCA on semipolar and volatile chemicals of $Z$. jujuba seeds. As most of bioactive compounds are present in the methanolic extract of $Z$. jujuba seeds, the UPLC-MS/MS results presented more information on

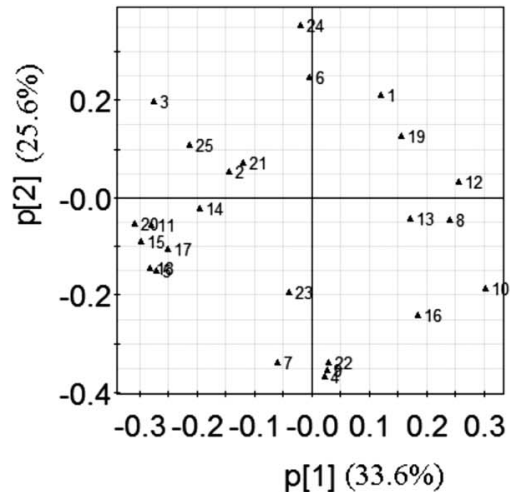

Loading plot (A)

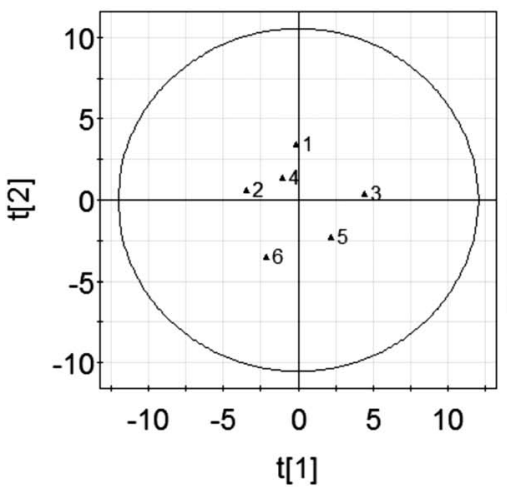

Score plot (A)

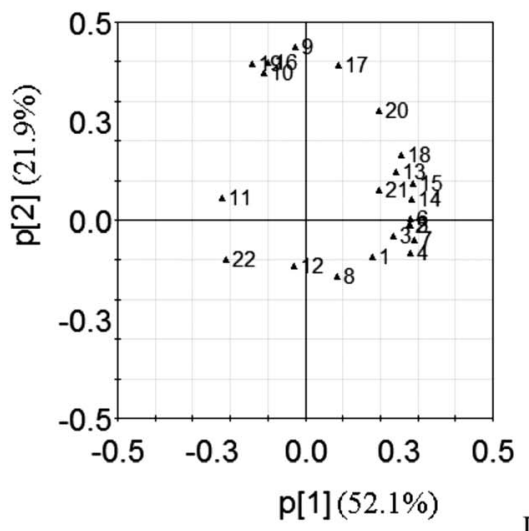

Loading plot (B)

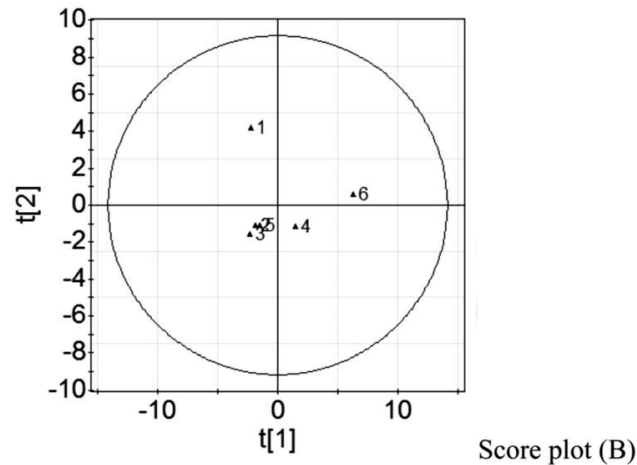

Fig. 3 Plots of PCA on semi-polar and volatile chemicals of Z. jujuba seeds. Loading plot $(A)$ and score plot $(A)$ are the results of semi-polar chemicals; loading plot (B) and score plot (B) are the results of volatile chemicals. 
Table 3 Component matrix of PCA for the semi-polar phytochemicals of $Z$. jujuba seeds

\begin{tabular}{|c|c|c|}
\hline \multirow[b]{2}{*}{ Chemical number $^{a}$} & \multicolumn{2}{|c|}{ Components } \\
\hline & 1 & 2 \\
\hline 1 & -0.342 & -0.534 \\
\hline 2 & 0.418 & -0.141 \\
\hline 3 & 0.803 & -0.502 \\
\hline 4 & -0.065 & 0.927 \\
\hline 5 & 0.787 & 0.370 \\
\hline 6 & 0.004 & -0.631 \\
\hline 7 & 0.177 & 0.856 \\
\hline 8 & -0.702 & 0.115 \\
\hline 9 & -0.084 & 0.891 \\
\hline 10 & -0.880 & 0.472 \\
\hline 11 & 0.812 & 0.141 \\
\hline 12 & -0.746 & -0.084 \\
\hline 13 & -0.499 & 0.114 \\
\hline 14 & 0.591 & 0.055 \\
\hline 15 & 0.863 & 0.228 \\
\hline 16 & -0.538 & 0.613 \\
\hline 17 & 0.722 & 0.269 \\
\hline 18 & 0.814 & 0.362 \\
\hline 19 & -0.447 & -0.321 \\
\hline 20 & 0.895 & 0.133 \\
\hline 21 & 0.347 & -0.179 \\
\hline 22 & -0.089 & 0.855 \\
\hline 23 & 0.120 & 0.487 \\
\hline 24 & 0.058 & -0.898 \\
\hline 25 & 0.622 & -0.272 \\
\hline
\end{tabular}

${ }^{a}$ Represents the chemicals having the same number listed in Table 1.

Table 4 Component matrix of PCA for the volatile chemicals of Z. jujuba seeds

\begin{tabular}{lrr}
\hline & Component & \\
\cline { 2 - 3 } Chemical number $^{a}$ & 1 & 2 \\
\hline 1 & & -0.199 \\
2 & 0.608 & -0.026 \\
3 & 0.942 & -0.090 \\
4 & 0.801 & -0.177 \\
5 & 0.949 & -0.028 \\
6 & 0.945 & 0.008 \\
7 & 0.950 & -0.109 \\
8 & 0.988 & -0.310 \\
9 & 0.286 & 0.963 \\
10 & -0.090 & 0.817 \\
11 & -0.374 & 0.121 \\
12 & -0.745 & -0.250 \\
13 & -0.105 & 0.267 \\
14 & 0.815 & 0.113 \\
15 & 0.963 & 0.199 \\
16 & 0.969 & 0.878 \\
17 & -0.338 & 0.860 \\
18 & 0.299 & 0.365 \\
19 & 0.869 & 0.867 \\
20 & -0.475 & 0.610 \\
21 & 0.661 & 0.168 \\
22 & 0.667 & -0.218
\end{tabular}

${ }^{a}$ Represents the chemicals having the same number listed in Table 2. quality. It was better to judge the quality on the basis of UPLC-MS/ MS analyses, and the GC-MS results could also be used as a supplementary reference. Fig. 3A shows the characteristic compounds in each sample. The first two principal components explained most of the total variance. It was surprising that betulinic acid had the leading peak in the UPLC-MS/MS profile. However, it was not the representative chemical in the first two principal components due to the insignificant variance. Only those chemicals having significant variance are recorded in the PCA model. The loading plot showed that ceanothic acid, $6^{\prime \prime}$-feruloylspinosin, jujuboside B (second quadrant), glycerol ester 1, linoleic acid derivative, pseudolaroside B (third quadrant), kaempferol 3-rutinoside, linoleic acid (fourth quadrant) are the principal components which defined the quality differences between $Z$. jujuba seeds. The sample located in a quadrant of score plot is characterized by high amounts of bioactive compounds located in the same quadrant and low amounts of compounds in the opposite quadrant of the loading plot. In this work, samples 1\#, 2\# and 4\# shared closely related similar qualities, which had high amounts of linoleic acid and kaempferol 3-rutinoside, and low amounts of ceanothic acid. However, samples 3\#, 5\# and 6\# showed significant differences between one another.

\section{Concluding remarks}

From the above results, it could be concluded that determination of the phytochemical profile is an efficient way to define the pharmaceutical quality of $Z$. jujuba seeds. By using analyses of UPLC-MS/MS and GC-MS, the semi-polar and volatile chemicals were identified and their levels were statistically compared. Betulinic acid was the leading semi-polar chemical in Z. jujuba seeds, and oleic acid was the leading volatile chemical. PCA could differentiate well between the quality of $Z$. jujuba seed samples.

\section{Acknowledgements}

We are grateful for financial support from the Guangdong Natural Science Funds for Distinguished Young Scholar, the National Natural Science Foundation of China (no. 31071638), the Knowledge Innovation Program of Chinese Academy of Sciences (no. KSCX2-EW-J-28), the Youth Innovation Promotion Association of Chinese Academy of Sciences, and the International Foundation for Science (no. F/4451-2).

\section{References}

1 J. J. Liu, B. Chen and S. Z. Yao, Talanta, 2007, 71, 668-675.

2 A. M. Pawlowska, F. Camangi, A. Bader and A. Braca, Food Chem., 2009, 112, 858-862.

3 C. H. Shou, Z. Y. Feng, J. Wang and X. X. Zheng, Planta Med., 2002, 68, 799-803.

4 D. Wheatley, J. Psychopharmacol., 2005, 19, 414-421.

5 B. Yang, F. Chen, Y. L. Hua, S. S. Huang, S. Lin, L. R. Wen and Y. M. Jiang, Food Chem., 2012, 131, 508-512.

6 Q. Q. Zhu, Y. M. Jiang, S. Lin, L. R. Wen, D. Wu, M. M. Zhao, F. Chen, Y. X. Jia and B. Yang, Biomacromolecules, 2013, 14, 1999-2003. 
7 Y. F. Sun, C. K. Song, H. Viemstein, F. Unger and Z. S. Liang, Food Chem., 2013, 138, 1998-2007.

8 Y. Wu, F. He, Q. Pan, Y. Shi, Z. D. Min and J. Y. Liang, Chem. Nat. Compd., 2011, 47, 369-372.

9 S. M. Lee, B. S. Min, C. G. Lee, K. S. Kim and Y. H. Kho, Planta Med., 2003, 69, 1051-1054.

10 S. Bernillon, B. Biais, C. Deborde, M. Maucourt, C. Cabasson, Y. Gibon, T. H. Hansen, S. Husted, R. C. H. de Vos, R. Mumm, H. Jonker, J. L. Ward, S. J. Miller, J. M. Baker, J. Burger, Y. Tadmor, M. H. Beale, J. K. Schjoerring, A. A. Schaffer, D. Rolin, R. D. Hall and A. Moing, Metabolomics, 2013, 9, 57-77.

11 B. Yang, Y. M. Jiang, M. M. Zhao, F. Chen, R. Wang, Y. L. Chen and D. D. Zhang, Food Chem., 2009, 115, 609-614.

12 B. Rhourri-Frih, P. Chaimbault, B. Claude, C. Lamy, P. Andre and M. Lafosse, J. Mass Spectrom., 2009, 44, 71-80.

13 F. Modugno, E. Ribechini and M. P. Colombini, Rapid Commun. Mass Spectrom., 2006, 20, 1787-1800.

14 A. M. Ayatollahi, M. Ghanadian, S. Afsharypour, O. M. Abdella, M. Mirzai and G. Askari, Iran. J. Pharm. Res., 2011, 10, 287-294.

15 H. M. Mukhtar, S. H. Ansari, M. Ali, T. Naved and Z. A. Bhat, Pharm. Biol., 2005, 43, 392-395.

16 W. Wang, J. Luo and L. Kong, China J. Chin. Mater. Med., 2009, 34, 2768-2773.

17 S. Guo, J. A. Duan, Y. P. Tang, Y. F. Qian, J. L. Zhao, D. W. Qian, S. L. Su and E. X. Shang, J. Pharm. Biomed. Anal., 2011, 56, 264-270.

18 A. Mari, D. Lyon, L. Fragner, P. Montoro, S. Piacente, S. Wienkoop, V. Egelhofer and W. Weckwerth, Metabolomics, 2013, 9, 599-607.
19 C. Besada, G. Sanchez, A. Salvador and A. Granell, Metabolomics, 2013, 9, 157-172.

20 J.-X. Cao, Q.-Y. Zhang, S.-Y. Cui, X.-Y. Cui, J. Zhang, Y.-H. Zhang, Y.-J. Bai and Y.-Y. Zhao, J. Ethnopharmacol., 2010, 130, 163-166.

21 L.-E. Wang, Y.-J. Bai, X.-R. Shi, X.-Y. Cui, S.-Y. Cui, F. Zhang, Q.-Y. Zhang, Y.-Y. Zhao and Y.-H. Zhang, Pharmacol., Biochem. Behav., 2008, 90, 399-403.

22 L. E. Wang, X. Y. Cui, S. Y. Cui, J. X. Cao, J. Zhang, Y. H. Zhang, Q. Y. Zhang, Y. J. Bai and Y. Y. Zhao, Phytomedicine, 2010, 17, 404-409.

23 Y. Ma, H. Han, S.-Y. Nam, Y.-B. Kim, J.-T. Hong, Y.-P. Yun and K.-W. Oh, J. Ethnopharmacol., 2008, 117, 318324.

24 L. C. Klein Junior, S. F. de Andrade and V. Cechinel Filho, Chem. Biodiversity, 2012, 9, 181-209.

25 Y. Jine, M. Lis, M. Szczypka and B. Obminska-Mrukowicz, Pol. J. Vet. Sci., 2012, 15, 305-313.

26 Z. Dang, W. Lai, K. Qian, P. Ho, K.-H. Lee, C.-H. Chen and L. Huang, J. Med. Chem., 2009, 52, 7887-7891.

27 J. Zhao, S. P. Li, F. Q. Yang, P. Li and Y. T. Wang, J. Chromatogr., A, 2006, 1108, 188-194.

28 J. I. Yoon, S. M. Al-Reza and S. C. Kang, Food Chem. Toxicol., 2010, 48, 1350-1354.

29 S. M. Al-Reza, J. I. Yoon, H. J. Kim, J. S. Kim and S. C. Kang, Food Chem. Toxicol., 2010, 48, 639-643.

30 M. K. Park, I. H. Cho, S. Lee, H. K. Choi, D. Y. Kwon and Y. S. Kim, Food Chem., 2010, 122, 1313-1319.

31 J. Yuk, K. L. McIntyre, C. Fischer, J. Hicks, K. L. Colson, E. Lui, D. Brown and J. T. Arnason, Anal. Bioanal. Chem., 2013, 405, 4499-4509. 